\title{
Mode Converter Optical Isolator Based on Dual Negative Refraction Photonic Crystal
}

\author{
Walid Aroua, Fathi AbdelMalek, Shyqyri Haxha, Senior Member, IEEE, Sintayehu Tesfa, and Habib Bouchriha
}

\begin{abstract}
A new design of an optical isolator based on photonic transitions in the interbands of a honeycomb structure that generates a dual negative refraction in a photonic crystal is presented. The involved photonic transition is associated to the perturbation of the dielectric constant of the medium. The band structure is determined using the plane wave method where the transmission spectra, field profile, and mode amplitudes are obtained by applying the finite difference time domain method. Due to the time-dependent perturbation of the refractive index of the medium that constitutes the dual negative refraction, asymmetric transmission mechanism is achieved for one of the desired modes, demonstrating optical isolation. Using the dual negative refraction effect in photonic crystal structure, the optical isolation is reported for only one of the desired optical modes. It is anticipated that the proposed mode conversion mechanism can be employed for designing ultrahigh-speed optical interconnections. The proposed optical isolator model is expected to have a significant impact on designing ultrahigh-speed integrated optical platforms.
\end{abstract}

Index Terms-Photonic crystals, metamaterials, nanostmctured materials, photonic integrated circuits, optical computing.

\section{INTRODUCTION}

O PTICAL computing and signal processing require isolation of light in optical components that can be achieved by a nonreciprocal light propagation. Non-reciprocal light propagation has been studied recently, where isolating optical signals that propagate in the accompanying photonic structures are reported [1]-[8]. Since optical isolators allow light to propagate predominantly in one direction while the signal that propagates in the opposite direction would be blocked or diverted, it prevents the ensued interference from happening [1]. In practice, the isolation of light in an optical component requires time-reversed symmetry breaking. It has

Manuscript received January 15, 2014; revised May 14, 2014; accepted June 10, 2014. Date of publication June 12, 2014; date of current version July 2, 2014.

W. Aroua and F. AbdelMalek are with the Department of Physics, Quantum Physics and Photonics Group, Faculty of Science, El Manar University, Tunis 2060, Tunisia, and also with the Department of Physics, University of Jazan, Jazan 45 142, Saudi Arabia (e-mail: waroua@yahoo.com; fathi.amalek@gmail.com).

S. Haxha is with the Department and Computer Science and Technology, University of Bedfordshire, Luton LU1 3JU, U.K. (e-mail: shyqyri.haxha@beds.ac.uk).

S. Tasfa is with the National Institute of Applied Sciences and Technology, Carthage University, Tunis 2060, Tunisia (e-mail: swmariam@jazanu.edu.sa).

H. Bouchriha is with the Department of Physics, Quantum Physics and Photonics Group, Faculty of Science, El Manar University, Tunis 2060, Tunisia (e-mail: habib.bouchriha@fst.rnu.tn).

Color versions of one or more of the figures in this paper are available online at http://ieeexplore.ieee.org.

Digital Object Identifier 10.1109/JQE.2014.2330762 been shown earlier that optical isolation can be achieved for instance by applying opto-acoustic effects [9], nonlinear materials and materials containing magneto-optic effects driven by external magnetic fields [10]-[12]. The optical isolators designed in these conventional approaches require large magnetic fields, however for small optical structures it is technically demanding to achieve isolation following these approaches. Hence, currently, several efforts have been geared towards avoiding the application of magneto-optic effects and achieve a complete optical isolation of the light in micro meter scale [13]. Recently, it has been shown that the use of photonic crystals (PCs) opens up to novel ideas and techniques to realize an optical isolator where the light that propagates in the backward direction is blocked or diverted [1]. The obtained optical isolator can be used primarily in optical interconnection which requires a nonreciprocal propagation of the light between different chips.

In this regard, we pursue to demonstrate an active optical isolator based in Dual-Negative Photonic Crystals (DNPC) structure for certain desired optical modes. In practical context, the working principle of DNPC structure is based on the fact that under certain conditions the electromagnetic wave can be dispersed and divided into two negatively refracted waves which lead to the Dual-Negative-Refraction (DNR) phenomena [13]. It is worth noting that in the DNR effect the overlap between two bands is necessary. This entails that the isolation of the light in the DNPC requires controlling of the transition of photons in the inter-sub band. Therefore, in order to realize this idea, the time dependent modulation of the refractive index is used. The DNPC with hexagonal lattices to generate the DNR effect is considered for practical reasons. Based on the geometrical symmetry of this structure, the lowest $\mathrm{TM}$ modes $\left(\mathrm{TM}_{2}\right.$ and $\left.\mathrm{TM}_{3}\right)$ can be generated by careful selection of the frequency of the incident modes. The proposed structure supports both propagating modes $\left(\mathrm{TM}_{2}\right.$ and $\mathrm{TM}_{3}$ ) in the forward direction, however in the opposite direction only the $\mathrm{TM}_{3}$ mode is propagating. In order to establish the isolation, the dielectric constant of the hexagonal rods is perturbed where the $\mathrm{TM}_{2}$ mode (desired mode) is blocked in the desired direction. With respect to the definition of the optical isolator introduced by Jalas et al [1] and Fan et al [2], we add an absorption filter centered at $\mathrm{TM}_{2}$ mode frequency in the output interface, where the dispersion of the optical modes is calculated by using the plane wave method. For The transmission spectra on the other hand, the field profile and the optical mode amplitudes, in both directions, are determined by employing the Finite Difference 


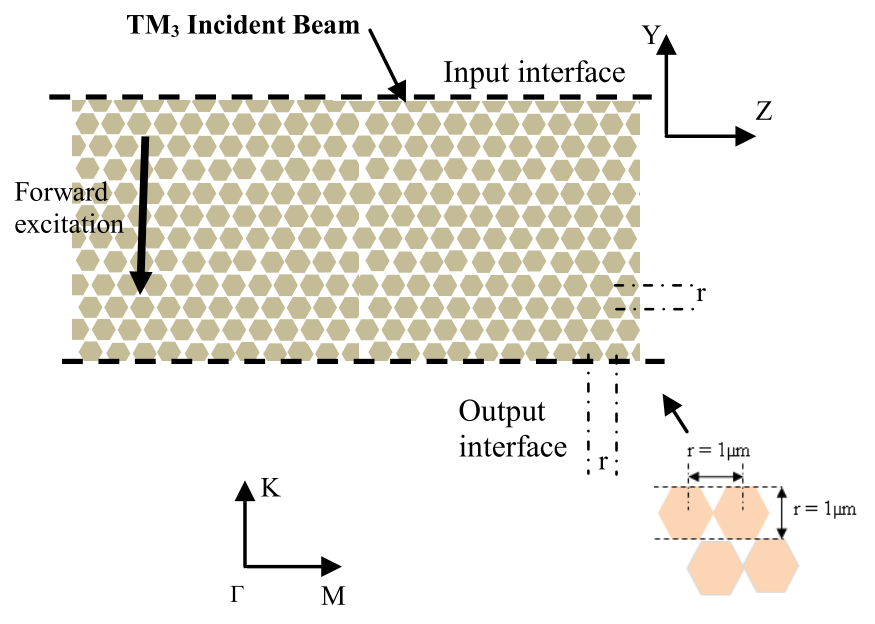

Fig. 1. Schematic representation of the mode converter optical isolator. The normal of the input interface is along $\Gamma \mathrm{M}$ direction. For convenience, the frequency and the wave vector are normalized by the lattice constant of the structure $a=1 \mu \mathrm{m}$. The rod dimension is fixed to around center to center distance $\mathrm{r}=0.5 \mu \mathrm{m}$.

Time Domain Method (FDTD). The FDTD is a powerful and versatile numerical method that is employed to solve various PCs complex structures [14].

Our simulation results show that the performance of the mode converter optical isolator, proposed in this study, has attained almost $100 \%$ at the operating wavelength of $1550 \mathrm{~nm}$ for the $\mathrm{TM}_{2}$ mode. In this regard, the proposed idea would have a great impact in transforming the information between various different integrated chips into a single platform or chip-to-chip integrated platforms.

\section{Mode Converter Optical Isolator}

A proposed mode converter optical isolator structure for a specific mode based on a negative photonic crystal (NPC) is illustrated in Fig. 1. The honeycomb lattice PC is constructed from hexagonal dielectric rods with $\varepsilon_{0}=11.56$, positioned in air with a filling ratio of $82.2 \%$. The rod dimension is fixed around center to center distance $\mathrm{r}=0.5 \mu \mathrm{m}$. It is envisaged with current advanced available technology that this structure can be realized by using holographic lithography formed by crystalline $\mathrm{Si}(\mathrm{c}-\mathrm{si})$. We choose to use this material due to its natural abundance and almost an ideal band gap [13].

In the present work, in order to induce mode conversions in the forward direction, we introduced temporal perturbation of the dielectric constant of the rods, whereas the considered modulation is expressed as

$$
\varepsilon=\varepsilon_{0}+\Delta \varepsilon
$$

where $\varepsilon_{0}=11.56$ is the dielectric constant of the nonperturbed structure and $\Delta \varepsilon$ represents the time dependent perturbation. It is worth pointing out that our device is a linear negative photonic crystal for which the Lorentz theorem reciprocity does not hold [1], therefore our proposed device can be used to realize an ideal optical isolator. The time dependence index is used to couple two specific propagation

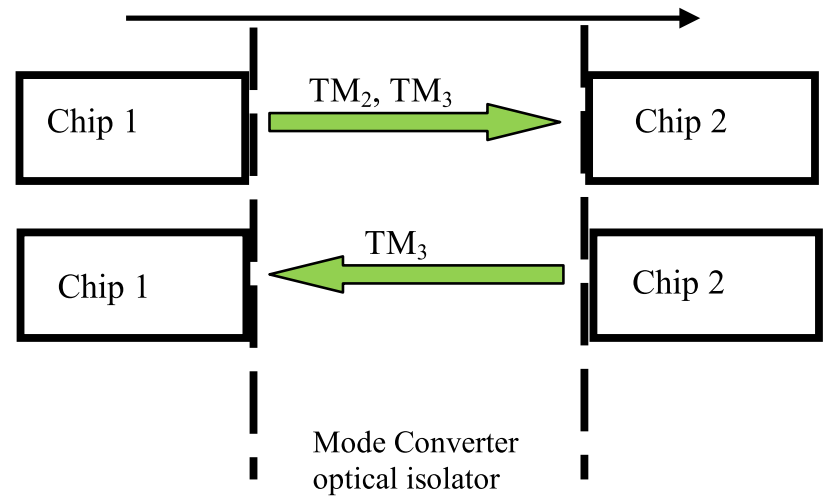

Fig. 2. Schematics of the nonreciprocal optical interconnection when the mode converter optical isolator structure is introduced between Chip1 and Chip 2.

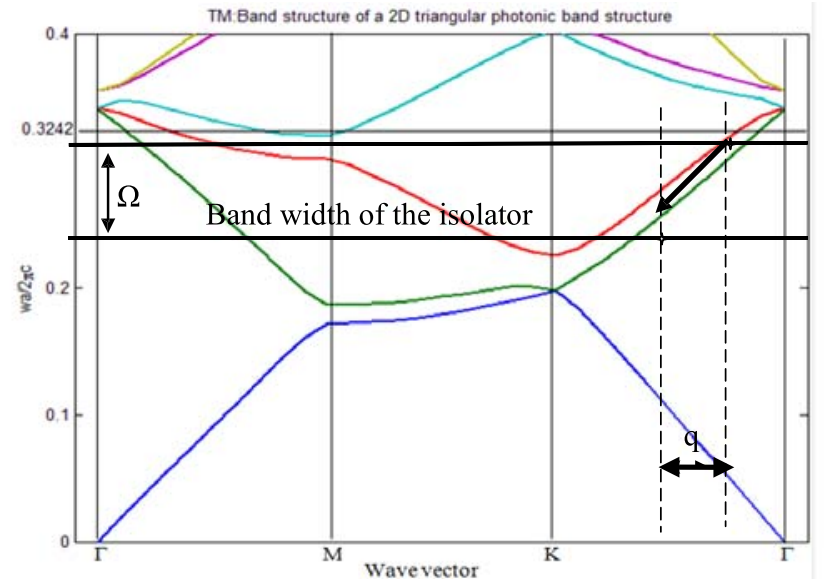

Fig. 3. Plots of the band structure of the unperturbed DNPC crystal. The bold arrow represents the optical transition between $\mathrm{TM}_{2}$ and $\mathrm{TM}_{3}$. The bandwidth of the isolator is designated by a maximum of the band overlap between $\mathrm{TM}_{2}$ (green curve) and $\mathrm{TM}_{3}$ (red curve) modes.

modes $\left(\mathrm{TM}_{2}\right.$ and $\mathrm{TM}_{3}$ modes) only in forward direction. By placing an absorbing filter centered at $\mathrm{TM}_{2}$ frequency in one direction, this structure can be used to realize a comprehensive optical isolator [2].

Moreover, the proposed system is envisaged to be applicable to create an interconnection between different optical chips. With this idea, Chip-to-chip possible optical interconnection using a proposed mechanism is depicted in Fig. 2, which shows that in the forward direction $\mathrm{TM}_{2}$ and $\mathrm{TM}_{3}$ can propagate from chip 1 to chip 2, but in the backward direction only $\mathrm{TM}_{3}$ is transmitted.

\section{Result And Discussion}

In order to calculate the photonic band structure, the plane wave method is employed where the electric field of the TM mode parallel to the rod axis is illustrated in Fig. 3.

From Fig. 3, it can be seen that the higher bands are overlapped specially for the second band $\left(\mathrm{TM}_{2}\right.$ mode) and third band ( $\mathrm{TM}_{3}$ mode). We notice that in the overlapping band region, the two bands are very close to each other, which is a key factor to design an ideal mode converter. In this regard, 
we demonstrate that the nonreciprocal light propagation in the NPC can be realized by inducing indirect transition in one direction in the considered photonic structure. In line with this, it has been shown elsewhere that when a photonic structure is subject to a refractive index modulation, the photon states can pass through inter band transitions [2].

In this study, in order to induce mode conversions, we choose to introduce a temporal perturbation of the dielectric constant of the rods, illustrated in Fig. 1. The perturbation can be generated in various ways. One particularly simple method is to exploit the nonlinear properties of one of the materials belonging to the crystal. This modulation allows photons that belong to different bands to go through inter band transitions, where shifts in frequency and momentum of the photon would be observed during the modulation process. On the other hand, an interband transition between two modes can be induced by modulating the rods with an additional dielectric perturbation which is given by

$$
\Delta \varepsilon=\delta(r) \cos (\Omega t-q r),
$$

where $\delta(r)$ is the modulation amplitude and $\Omega$ represents the modulation frequency,

$$
\Omega=\omega_{1}-\omega_{2},
$$

where, $\omega_{1}$ and $\omega_{2}$ represent normalized frequency of $\mathrm{TM}_{3}$ and $\mathrm{TM}_{2}$ modes, respectively. We notice that the phase needed in eq. (2) can be controlled by the modulation frequency $\Omega$. It is important to note, that the wave vector approximately satisfies the phase-matching condition,

$$
\Delta K=\left(K_{2}-K_{1}\right)-(-q) \approx 0,
$$

where $K_{1}$ and $K_{2}$ represent the wave vector for $\mathrm{TM}_{3}$ and $\mathrm{TM}_{2}$ modes, respectively.

It is worth also noting that the mode transition between the $\operatorname{TM}_{3}\left(\omega_{1}, \mathrm{k}_{1}\right)$ mode and the $\mathrm{TM}_{2}\left(\omega_{2}, \mathrm{k}_{2}\right)$ mode occurs only when the phase-matching condition is achieved. In our case, for $\mathrm{TM}_{3}$ mode this condition is only valid in the forward direction. From Fig. 3 , we notice that the mode at $\left(\omega_{1},-\mathrm{k}_{1}\right)$ is not phase-matched with any other modes of the NPC. It can also be seen that while the mode at $\left(\omega_{1}, \mathrm{k}_{1}\right)$ undergoes a photonic transition its' time-reversed counterpart at $\left(\omega_{1},-\mathrm{k}_{1}\right)$ is not affected. In this view the nonreciprocity of propagation of the light is key parameter to design an optical isolator, therefore in order to demonstrate the optical isolation, the frequency of the bandwidth is calculated where the band overlap between the $\mathrm{TM}_{2}$ and $\mathrm{TM}_{3}$ modes is at its maximum peak $\Omega=\omega_{1}-\omega_{2}=0.0042(\mathrm{a} / \lambda)$.

In our simulation, the width of the NPC slab is selected to be 8 periods. To induce transitions between the $\mathrm{TM}_{3}$ mode at $\omega_{1}=0.3242(a / \lambda)$ and the $\mathrm{TM}_{2}$ mode at $\omega_{2}=(0.3200 a / \lambda)$, the phase matching condition is $q=0.59 \mathrm{k}_{0}=k_{1}-k_{2}$ at the operating wavelength of $1.55 \mu \mathrm{m}$ (associated with $\mathrm{k}_{0}$ ). For the sake of convenience, the modulation amplitude $\delta(r)$ is kept fixed at around 0.251 . In order to optimize the length $(\mathrm{L})$ of the slab of the NPC structure, we simulate the modes amplitudes as a function of the propagation axis (z-axis), starting with 3 periods, and proceeding with one step until we reach the $13^{\text {th }}$ period.

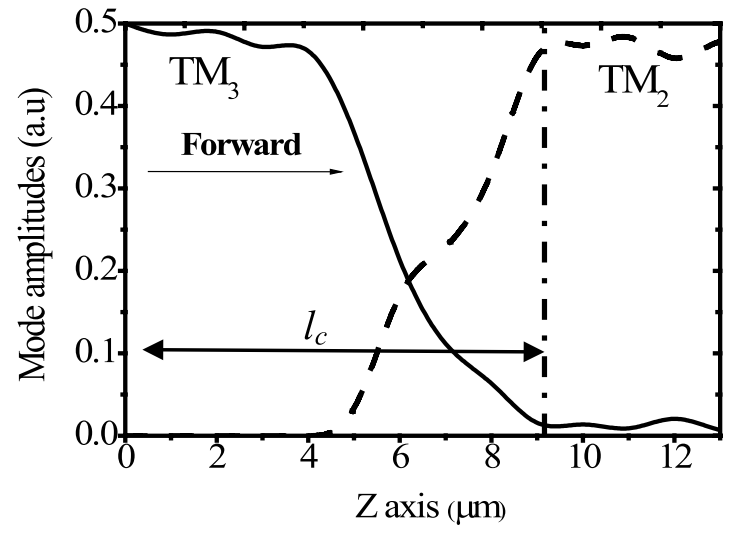

Fig. 4. Plots of the $\mathrm{TM}_{2}$ and $\mathrm{TM}_{3}$ mode amplitude in the forward directions against $Z$ axis, when the period is fixed to $1 \mu \mathrm{m}$, and $l_{c}$ represent the coherence length.

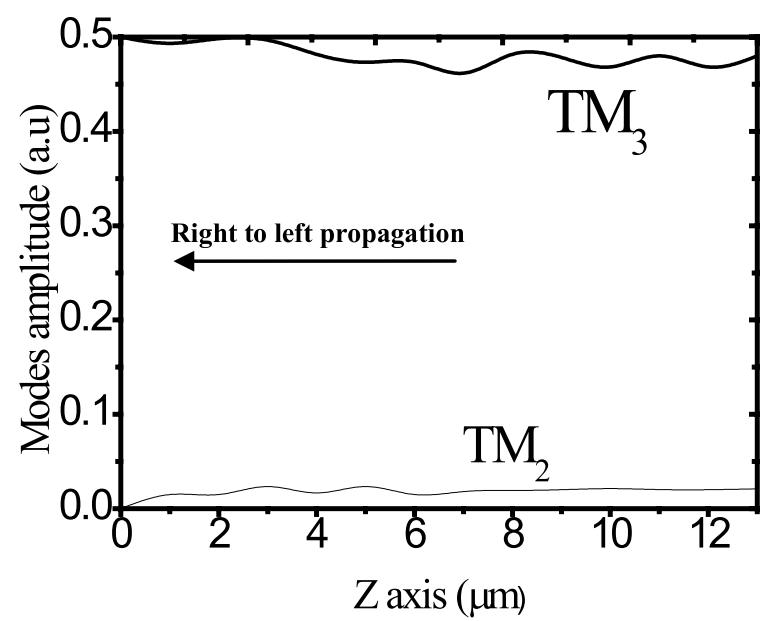

Fig. 5. Plots of the amplitude of the $\mathrm{TM}_{2}$ and $\mathrm{TM}_{3}$ modes when the light propagates in the backward direction.

As illustrated in Fig. 4, conversion of the modes is observed for the dielectrics periods from 1 to 4 . It can be clearly seen that in this particular region, only the $\mathrm{TM}_{3}$ mode can propagate through the NPC structure. However, between 4 to 9 periods, one can see that the amplitude of the $\mathrm{TM}_{2}$ mode increases whereas the amplitude of the $\mathrm{TM}_{3}$ decreases sharply, indicating that the modes start to be converted. In addition to this, for the regions greater than $9 \mu \mathrm{m}$ (note that the coherence length is defined to be $l_{\mathrm{c}}=9 \mu \mathrm{m}$ ), a complete mode conversion through the NPC slab is achieved. It is worth emphasizing that the coherence length $l, \mathrm{c}$ is the propagation distance necessary to observe the complete conversion of the modes.

Next, we realize a mode converter optical isolator for a particular mode $\left(\mathrm{TM}_{3}\right.$ mode) when the length (L) is fixed to around 9 periods. Fig. 5 shows the propagation of both modes $\mathrm{TM}_{2}$ and $\mathrm{TM}_{3}$ in the backward direction. It is clear from this figure that $\mathrm{TM}_{2}$ mode amplitude is constant, which is significantly small compared to that of the $\mathrm{TM}_{3}$. In other words, the amplitude of the $\mathrm{TM}_{3}$ mode is at a maximum, while the amplitude of the $\mathrm{TM}_{2}$ mode is nearly zero. The obtained results demonstrate that the photon from the $\mathrm{TM}_{3}$ mode can 
be converted to the $\mathrm{TM}_{2}$ mode, although only in the forward direction, still confirming that the proposed structure exhibits mode conversation optical isolator.

In the forward direction for a length (L) of the slab greater than the coherence length $L_{\mathrm{c}}$ [2], we observe a total mode conversion, where the resulting scattering matrix is calculated by;

$$
\left[\begin{array}{l}
T M_{3} \\
T M_{2}
\end{array}\right]=\left[\begin{array}{ll}
0 & 1 \\
1 & 0
\end{array}\right]\left[\begin{array}{l}
T M_{3} \\
T M_{3}
\end{array}\right]
$$

We notice that, this matrix is symmetric. It is worth mentioning that in order to create an optical isolator Fan et al. [2] introduced an absorption filter centered at $\mathrm{TM}_{2}$ mode frequency. This structure can absorb all of the light in one direction and the transmission matrix becomes;

$$
\left[\begin{array}{l}
T M_{3} \\
T M_{2}
\end{array}\right]=\left[\begin{array}{ll}
0 & 1 \\
0 & 0
\end{array}\right]\left[\begin{array}{l}
T M_{3} \\
T M_{3}
\end{array}\right]
$$

In this case, the transmission matrix is asymmetric and a complete optical isolation is obtained [15]-[21].

Furthermore, in Fig. 6a, we have illustrated the snapshot of the mode profile when a pulse with a Gaussian of the frequency $\omega_{1}$ is launched on the left hand side of the mode converter optical isolator structure. Here we use a waveguide source in order to study the propagation of light through the optical isolator [1]. One can see that for an incident angle of $\theta=30^{\circ}$, part of the $\mathrm{TM}_{3}$ mode is converted to $\omega_{2}$ $\left(\mathrm{TM}_{2}\right.$ mode). In this regard, photons are initially located in the mode $\mathrm{TM}_{3}$ and during propagation, in the NPC structure; they experience a transition process to the $\mathrm{TM}_{2}$ mode. In order to observe more details on the non-reciprocity of the light that leads to optical isolation, we reverse the source and excite the structure from the right hand side, the snap-shot is illustrated in Fig. 6a. As can be seen from this figure, in this direction only $\mathrm{TM}_{3}$ mode can propagate through the NPC; non-conversion of the mode is observed.

We notice that when exciting the slab by $\mathrm{TM}_{3}$ mode, we divert entire light in forward direction and the optical isolator is achieved according to the definition of $\mathrm{Yu}$ et al. [2] and Jallas et al. [1]. However, for convincing reasons we excite the DNPC slab by $\mathrm{TM}_{2}$ mode in the backward direction, and the obtained results are illustrated in Fig. 6c, indicating that the entire light has been blocked in backward direction.

Our simulations (Fig. 6c) demonstrate that the amplitude power of the propagated $\mathrm{TM}_{2}$ mode is less than 0.15 (a.u), in contrast the amplitude of the $\mathrm{TM}_{3}$ propagated mode is around 1 , consequently we conclude that this mode cannot propagate in the backward direction and the light is radiated. Fig. $6 \mathrm{c}$ shows that the light is blocked and the optical isolation phenomenon is demonstrated. In order to understand in more details the isolation effect, we calculate the transmission matrix, shown in Fig. 7, with $\mathrm{TM}_{2}$ mode in the backward direction using eq. (7); our calculations show that this matrix is asymmetric.

$$
\left[\begin{array}{l}
B_{1} \\
B_{2}
\end{array}\right]=\left[\begin{array}{ll}
0 & 0 \\
1 & 0
\end{array}\right]\left[\begin{array}{l}
A_{1} \\
A_{2}
\end{array}\right]
$$

In other words, as the $\mathrm{TM}_{3}$ mode propagates along the $+\mathrm{Z}$ direction, it starts to be converted into the $\mathrm{TM}_{2}$ mode .

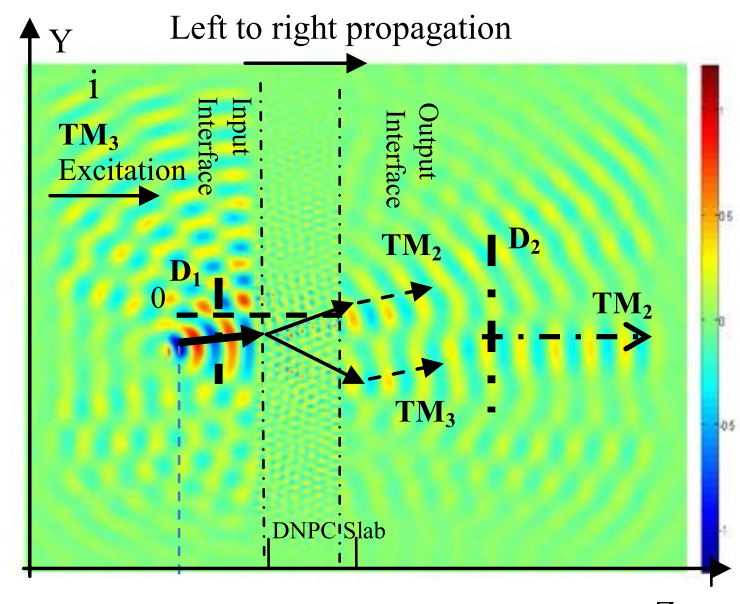

0

(a)

i

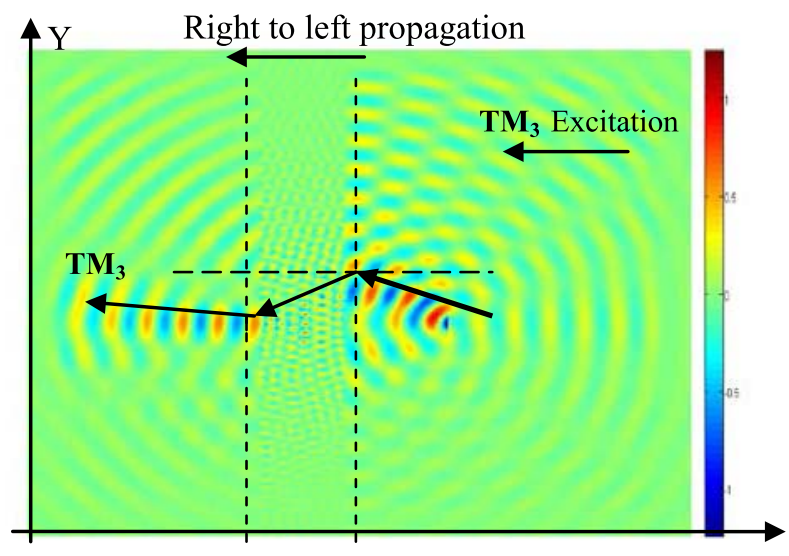

(b)

Z

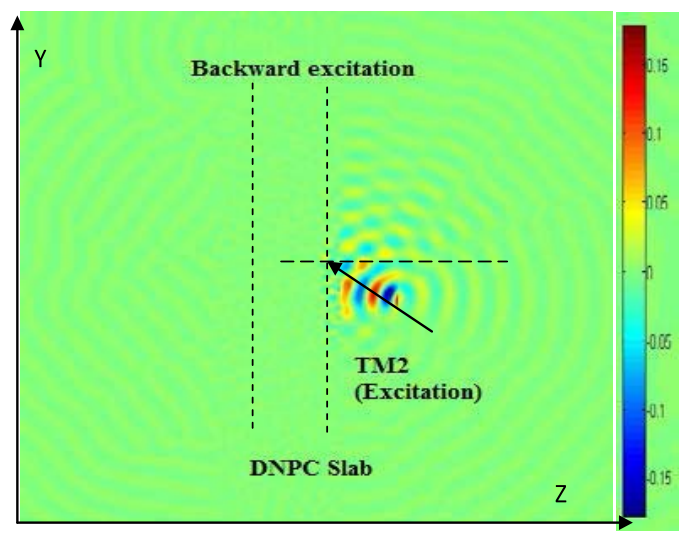

(c)

Fig. 6. Snap-shot of the distribution of the magnetic fields in $\mathrm{Z}$ direction at 20000 time steps in the forward (a) and backward (b) directions with $\omega a / 2 \pi c=0.3242(a / \lambda)$ for 8 periods of the dielectrics. The DNPC slab is excited with the incident angle $\theta=30^{\circ}$. (c) Snap-shot of the magnetic distribution field in $\mathrm{Z}$ direction at 20000 time steps in the backward direction for the $\mathrm{TM}_{2}$ mode for 7 periods of dielectrics. The DNPC slab is excited with incident angle $\theta=30^{\circ}$.

Calculation of the power at the source and the detector is also calculated. The incident $\left(P_{\text {inc }}\right)$ represents the ratio between the power at the detector $\left(\mathrm{D}_{1}\right)$ and the power at the source. In a similar way, the transmitted power $\left(P_{\text {trans }}\right)$ 


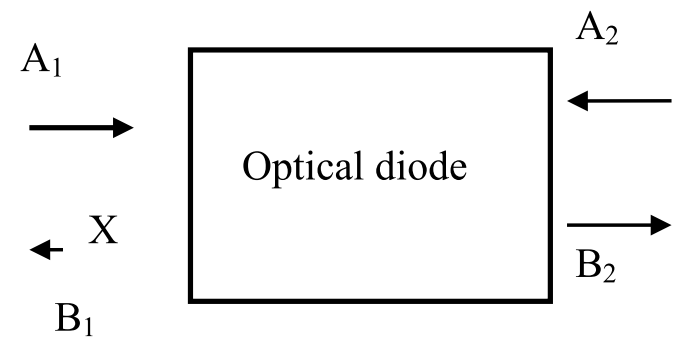

Fig. 7. Transmission matrix of $\mathrm{TM}_{2}$ mode in backward direction.
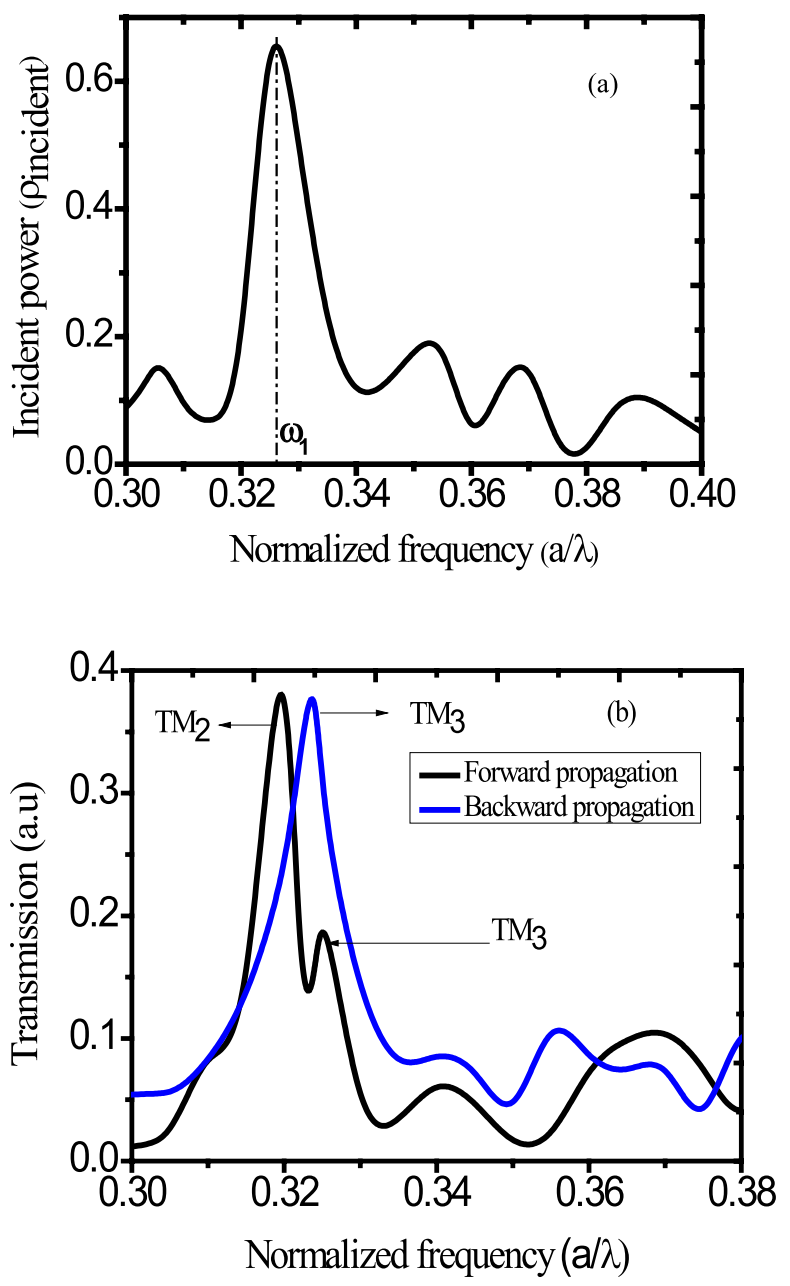

Fig. 8. (a): Plot of the incident power as function of the normalized frequency, when the maximum is fixed at $\omega_{1}=0.3242(\mathrm{a} / \lambda)$, (b): Plot of the transmission power as function of the normalized frequency in the forward and backward propagation, where $\omega_{1}$ represents the frequency of the $\mathrm{TM}_{3}$ mode, and $\omega_{2}$ presents the frequency of the $\mathrm{TM}_{2}$ mode.

represents the ratio between the power at the detector $\left(\mathrm{D}_{2}\right)$ and the power at the source.

In Fig. 8(a) and (b), we illustrated the incident and transmitted powers in the forward direction. It is clear from these figures that in the forward direction the $\mathrm{TM}_{3}$ mode is converted to $\mathrm{TM}_{2}$. From Fig. 8(b), it can also be seen that $70 \%$ of the incident power is converted, reaching its maximum at $0.32 \mathrm{a} / \lambda$. Thus, the incident and transmission powers as a function of the normalized frequency are shown in these figures. As can be seen from Fig. 8(b) the transmission spectra in the backward direction reached its maximum at 0.4 (a.u) where $\omega_{1}=0.32(a / \lambda)$. From this spectrum, we calculate the optical loss ratio (S) introduced by the NPC structure. We notice that in this direction, since no conversion of the mode is observed, the total loss in this direction is introduced by the mode converter isolator. By using the following definition

$$
S=\frac{\max \left(P_{\text {inciT M }}\right)-\max \left(P_{\text {transT M3 }}\right)}{\max \left(P_{\text {incT M } 3}\right)}
$$

where $\mathrm{S}$ is around $19 \%$ in the backward direction, we notice that most of the power is lost at the input interface of the NPC structure and from the diffusion of the medium. Nonetheless, it should be noted that the optical loss should be the same in both directions for the isolator.

In this design, we excite the $\mathrm{TM}_{3}$ mode in both directions, as can be seen, in the backward direction non conversion of the light is observed (blue line), nevertheless in the forward direction the entire light is converted to $\mathrm{TM}_{2}$ mode (black line).

From Fig. 8(b) one can clearly perceive the differences between the forward and backward light transmission behavior magnitudes. It can be observed that the asymmetric transmission is evident from the Fig. 8(b), and that the maximum transmission in the forward direction doesn't concede with that of the transmission in the backward direction which results in a spectra window of the bandwidth equal to $0.0042(\mathrm{a} / \lambda)$.

\section{CONCLUSION}

We have presented a novel mode converter optical isolator based on dual negative refraction photonic crystal structure where the nonreciprocal isolation is accomplished by temporal refractive index modulations introduced in the direction of the excitation. We have demonstrated the light propagation and optical mode behavior in the forward and backward directions. In the forward direction, we have demonstrated that $70 \%$ of the $\mathrm{TM}_{3}$ mode is converted to the $\mathrm{TM}_{2}$ mode, however in the backward direction $100 \%$ of the $\mathrm{TM}_{3}$ is transmitted. By using the DNR effect, we have selected and blocked only desired optical modes. It has been demonstrated that the maximum transmission in both directions is located at different frequencies, resulting in an optical isolation region. This study would play an important role in future optical integrating and interconnecting nano-optoelectronic devices which still remain a challenging task that the nanotechnology industry is facing.

\section{REFERENCES}

[1] D. Jalas et al., "What is-And what is, not-An optical isolator," Nature Photon., vol. 7, no. 8, pp. 579-582, 2013.

[2] Z. Yu and S. Fans, "Complete optical isolation created by indirect interband photonic transitions," Nature Photon. Lett., vol. 3, no. 2, pp. 91-94, Feb. 2009.

[3] J. Koch, A. A. Houck, K. Le Hur, and S. M. Givin, "Time-reversalsymmetry breaking in circuit-QED-based photon lattices," Phys. Rev. A, vol. 82, p. 043811 , Oct. 2010.

[4] L. Feng et al., "Nonreciprocal light propagation in a silicon photonic circuit," Science, vol. 333, no. 6043, pp. 729-733, Aug. 2011.

[5] K. Fang, Z. Yu, and S. Fan, "Photonic Aharonov-Bohm effect based on dynamic modulation," Phys. Rev. Lett., vol. 108, pp. 153901-153906, Apr. 2012.

[6] H. Lira, Z. Yu, S. Fan, and M. Lipson, "Electrically driven nonreciprocity induced by interband photonic transition on a silicon chip," Phys. Rev. Lett., vol. 109, no. 3, pp. 033901-033905, Jul. 2012. 
[7] S. F. Preble, J. T. Robinson, S. Manipatruni, and M. Lipson, "Farfield control of radiation from an individual optical nanocavity: Analogue to an optical dipole," Phys. Rev. Lett., vol. 100, no. 4, pp. 043902-1-043902-4, Jan. 2008.

[8] S. F. Preble, Q. Xu, and M. Lipson, "Changing the colour of light in a silicon resonator," Nature Photon., vol. 1, no. 5, pp. 293-296, May. 2007.

[9] M. S. Kang, A. Butsch, and P. St. J. Russell, "Reconfigurable lightdriven opto-acoustic isolators in photonic crystal fibre," Nature Photon., vol. 5, no. 9, pp. 549-553, 2011.

[10] Y. Shoji, K. Mitsuya, and T. Mizumoto, "Silicon-based magneto-optical isolator and circulator fabricated by direct bonding technology," in Proc. Prog. Electromagn. Res. Symp., Stockholm, Sweden, Aug. 2013, pp. 21-24.

[11] K. Fang, Z. Yu, V. Liu, and S. Fan, "Ultracompact nonreciprocal optical isolator based on guided resonance in a magneto-optical photonic crystal slab," Opt. Lett., vol. 36, no. 21, pp. 4254-4256, Nov. 2011.

[12] M.-C. Tien, T. Mizumoto, P. Pintus, H. Kromer, and J. E. Bowers, "Silicon ring isolators with bonded nonreciprocal magneto-optic garnets," Opt. Exp., vol. 19, no. 12, pp. 11740-11745, Jun. 2011.

[13] G. Y. Dong, J. Zhou, X. L. Yang, and L. Z. Cai, "Dual-negative refraction in photonic crystals with hexagonal lattices," Opt. Exp., vol. 19, no. 13, pp. 12119-12124, Jun. 2011

[14] S. K. Yee, "Numerical solution of initial boundary value problems involving Maxwell's equations in isotropic media," IEEE Trans. Antennas Propag., vol. 13, no. 6, pp. 1135-1146, Jun. 1966.

[15] M. J. Lockyear, A. P. Hibbins, K. R. White, and J. R. Sambles, "One-way diffraction grating," Phys. Rev. E, vol. 74, no. 5, p. 056611, Nov. 2006

[16] A. Mandatori, M. Bertolotti, and C. Sibilia, "Asymmetric transmission of some two-dimensional photonic crystals," J. Opt. Soc. Amer. B, vol. 24 no. 3, pp. 685-690, Mar. 2007.

[17] A. E. Serebryannikov, "One-way diffraction effects in photonic crystal gratings made of isotropic materials," Phys. Rev. B, vol. 80, no. 15, p. 155117 , Oct. 2009.

[18] X. F. Li, X. Ni, L. Feng, M. H. Lu, C. He, and Y. F. Chen, "Tunable unidirectional sound propagation through a sonic-crystal-based acoustic diode," Phys. Rev. Lett., vol. 106, no. 8, p. 084301, Feb. 2011.

[19] A. E. Serebryannikov, A. O. Cakmak, and E. Ozbay, "Multichannel optical diode with unidirectional diffraction relevant total transmission," Opt. Exp., vol. 20, no. 14, pp. 14980-14990, Jul. 2012.

[20] S. Xu, C. Qiu, and Z. Liu, "Acoustic transmission through asymmetric grating structures made of cylinders," J. Appl. Phys., vol. 111, no. 9, p. 094505, 2012.

[21] A. E. Serebryannikov, E. Ozbay, and S. Nojima, "Asymmetric transmission of terahertz waves using polar dielectrics," Opt. Exp., vol. 22, no. 3, pp. 3075-3088, Feb. 2014.

Walid Aroua received the Ph.D. degree in quantum physics from the University of El Manar, Tunis, Tunisia, in 2005, with an excellent rating. Since 2006, he has been an Assistant Professor of Physics with the National Institute of Applied Science and Technology, University of Cartage, Tunis. He is a research expertise focusing on developing novel photonic nanostructures to design all-optical platforms. His research activity is concentrated mainly in the field of quantum optics.
Fathi AbdelMalek received the Ph.D. degree from the Ecole Centrale de Lyon, Lyon, France, in 1999. In this Ph.D. dissertation, he developed models and numerical schemes for the study of both periodic structures and rare earth ions' effects on the propagation of optical signals in forward and backward directions. He was a Research Associate and a Research Fellow at various U.S. and U.K. universities, where he carried out research on photonic crystals, grating for telecommunication, and photonics. Since 2011, he has been a Ful Professor with the Department of Physics and Engineering, National Institute of Applied Sciences and Technology, University of Carthage, Tunis, Tunisia. His current research interests include nanophotonics, nanoscale materials, microstructured fibers, and quantum optics.

Shyqyri Haxha received the M.Sc. and Ph.D. degrees from City University London, London, U.K., in 2000 and 2004, respectively. He has received several world class industrial E.M.B.A. and M.B.A. diplomas. In 2014, he joined the Computer Science and Technology, University of Bradfordshire, Luton, U.K., as a Senior Lecturer in Electronic Engineering. Prior to this post, he was a Lecturer in Optic Communication with the Department of Electronics, Kent University, Canterbury, U.K. His current research interests include photonic crystal devices, metamaterials, biosensors, photonic crystal fibers, surface plasmon polaritons, high-speed modulators, OCDMA, and OMIMO systems. He was a recipient of the SIM Post-Graduate Award from the Worshipful Company of Scientific Instrument Makers, Cambridge, U.K., for his highly successful contribution in research in 2003. He has been a Keynote Speaker of numerous world class conferences.

Sintayehu Tesfa received the Ph.D. degree in quantum optics from Addis Ababa University, Addis Ababa, Ethiopia, in 2008. He is currently an Assistant Professor of Physics at Jazan University, Jazan, Saudi Arabia. He was a Guest Scientist with the Max Planck Institute, where he was involved in complex system in quantum optics for one year. His research expertise is in the area of theoretical quantum optics and information, and active plasmonics.

Habib Bouchriha received the Ph.D. degree from the Ecole Normale Superieure de Physique, Paris, France, in 1972. From 1972 to 1982, he conducted research in solid-state physics at the Centre National de Recherche Scientifique, Paris. He was a recipient of the Best Physicist Prize at the Mediterranean in 1995. Since 1984, he has been leading the Condensed Matter Laboratory at the Faculty of Science, University of El Manar, Tunis, Tunisia. He has a track record of around 160 journal papers, many of them in Physics Reviews (A, B) and the IEEE. 